\title{
Die Geschichte des Vergewaltigungstatbestandes - Aufbruch contra Konservierung
}

\section{Einführung}

Die Auseinandersetzung mit $\S 177$ StGB, dem Vergewaltigungstatbestand, erfordert Geschichtsbewusstsein: Rechtsprechung und Wissenschaft zu $§ 177$ StGB erschließen sich nur, wenn der historischen Entwicklung nachgegangen wird. Dabei sind insbesondere der Begriff der Bescholtenheit, das Tatbestandsmerkmal der Gewalt und das Institut des minder schweren Falls von Bedeutung. Wie kein anderer Tatbestand ist $\S 177$ StGB Ausdruck traditioneller, patriarchal geprägter Rollenbilder über Frauen und Männer. Erst $^{1}$ das 33. StÄG von $1997^{2}$ und das 6. StrRG von $1998^{3}$ setzten dringende Reformanliegen um und konnten Strafbarkeitslücken schließen. Das Verständnis von einer Vergewaltigungstat hat sich jedoch nicht grundlegend gewandelt. Es finden sich weiterhin Relikte überkommener Vergewaltigungsmythen. Vergewaltigungsspezifische Auslegungstraditionen werden konserviert.

Der folgende Beitrag beschäftigt sich zuvörderst mit der Frau als Vergewaltigungsopfer. Dadurch soll nicht geleugnet werden, dass Männer sexueller Gewalt ausgesetzt sind. Diese Vorgehensweise ist vielmehr dem Umstand geschuldet, dass Männer erst seit 1997 in den Opferkreis des $\S 177$ StGB miteinbezogen und Rechtsprechung und Wissenschaft von weiblichen Opferkonstellationen geprägt sind. ${ }^{4}$

\section{Den Vergewaltigungstatbestand prägende außerrechtliche Vorstellungen}

Die Wurzeln der den $\S 177$ StGB prägenden Auffassungen liegen in den patriarchalen Strukturen, die in der Gesellschaft und damit auch in den Gesetzen verankert sind. Erst ab Ende der 1960iger Jahre zeichnet sich ein Veränderungsprozess hinsichtlich der sexuellen Moral und der Geschlechterrollen ab. Erinnert sei in diesem Zusammenhang daran, dass der Gleichberechtigungsgrundsatz aus Art. 3 II GG ${ }^{5}$ erst $1977^{6}$ vollständig im Familienrecht umgesetzt und damit zumindest formal die gesetzgeberische Gleichstellung des Mannes und der Frau im Rahmen der ehelichen Lebensgemeinschaft bewirkt wurde. In einer patriarchalen Gesellschaft werden Männer und Frauen mit Eigen-

1 Von 1871 bis 1997 erfuhr der Tatbestand des § 177 StGB nur marginale Veränderungen; dazu Sick, Sexuelles Selbstbestimmungsrecht und Vergewaltigungsbegriff, 1993, S. $50 \mathrm{ff}$.

2 BGBl. I 1607; vgl. zur Reform u. a. Renzikowski, NStZ 1999, 377 ff., 440 ff.

3 BGBl. I 164.

4 Laut der bundesweiten PKS von 2007 sind Täter von Vergewaltigungen und sexuellen Nötigungen zu $99 \%$ bzw. zu $98 \%$ männlich, $1 \%$ bzw. $2 \%$ sind weiblich. Opfer sind zu 95,5\% bzw. zu 91,9\% weiblich, zu 4,5\% und 8,1\% männlich.

5 Elisabeth Selbert bewirkte dessen Existenz; dazu Böttger, Das Recht auf Gleichheit und Differenz, 1. Auflage (1990).

6 1. EheRG vom 14.6.1976 (BGBl I S. 1421); vgl. Palandt-Diederichsen, 39. Auflage (1980), Einf. V. $§ 1353$ Ziff. 6. 
schaften belegt, die sich eindeutig aus biologischen Unterschieden, ${ }^{7}$ aus (christlichen) Naturrechtslehren und aus jahrhundertealten Traditionen ergeben sollen. ${ }^{8}$ Das Gutachten des I. Zivilsenats des BGH ${ }^{9}$ von 1954 zur Struktur der Familie sowie das Urteil des BVerfG $^{10}$ zur Strafbarkeit der Homosexualität von 1957 bieten dafür anschauliche Beispiele. Das Wesen der Frau ist demnach unter anderem von Schwäche, Hingebung und Schamhaftigkeit gekennzeichnet. Dem Mann zugeschrieben werden demgegenüber unter anderem die Attribute des Durchsetzungsvermögens und der Selbständigkeit. Mannsein bedeutet, aktiv - auch unter Einsatz von physischer Gewalt - vorzugehen, Frausein, die passive Rolle einzunehmen. ${ }^{11}$ Eine ,anständige“ Frau erlangt ihre sittliche Vollkommenheit durch die Eingehung der Ehe und muss sich - im Gegensatz zum Mann ${ }^{12}$ - bis dahin ihre Jungfräulichkeit bewahren. Mit der Eheschließung verpflichten sich Frau und Mann zum regelmäßigen Geschlechtsverkehr (§ 1353 I BGB) ${ }^{13}$ dessen Vornahme eine der tragenden Säulen der Ehe darstellt. ${ }^{14}$ Das unbescholtene Mädchen, ${ }^{15}$ die liebende und hingebungsvolle Ehefrau und Mutter sowie der tat- und wirkkräftige Mann erscheinen damit als gesellschaftliches Idealbild. Das Wesen der Frau und des Mannes und folglich auch deren Lebensgestaltung sind determiniert. Abweichungen erfuhren lange Zeit auch strafrechtliche Sanktionen, so dass die einschlägigen Normen aus sich heraus ein Abbild geschlechtsspezifischer Rollenzuschreibungen darstellten. ${ }^{16}$ Mit deren Abschaffung ging dieses Spiegelbild traditioneller Auffassungen verloren und es trat insofern zumindest eine strafrechtliche Liberalisierung ein. Ein durchgreifender Wandel der Auffassungen über Sexualität und das Geschlechterverhältnis war damit jedoch nicht verbunden. Dies zeigt insbesondere $\S 177 \mathrm{StGB}$, der durch das 4 . StrRG

7 Dazu Möbius, Über den physiologischen Schwachsinn des Weibes, 11.-15. Auflage (1977); Weininger, Geschlecht und Charakter, 21. Auflage (1920).

8 Dazu Greif/Schobesberger, Einführung in die Feministische Rechtswissenschaft, 2. Auflage (2007), S. 5 ff.

9 BGHZ 11, Anhang, I. Zivilsenat, S. $65 \mathrm{ff}$.

10 BVerfG 6, 389.

11 Greif/Schobesberger, Einführung in die Feministische Rechtswissenschaft, 2. Auflage (2007), S. $11 \mathrm{f}$.

12 Ebenso Hundertmark, Streit 1986, S. 55 ff., S. 56; vgl. Sonderegger, Die strafrechtliche Behandlung der Frau, 1923, S. $22 \mathrm{ff}$.

13 Palandt-Brudermüller, 69. Auflage (2010), § 1353 Rn. 5.

14 Dazu BGH NJW 1967, 1079: „Die Frau genügt ihren ehelichen Pflichten nicht schon damit, daß sie die Beiwohnung teilnahmslos geschehen läßt. Wenn es ihr ... versagt bleibt, im ehelichen Verkehr Befriedigung zu finden, so fordert die Ehe von ihr doch eine Gewährung in ehelicher Zuneigung und Opferbereitschaft und verbietet es, Gleichgültigkeit oder Widerwillen zur Schau zu tragen".

$15 \S 182$ StGB a. F. spiegelt dies wider, indem er die Verführung von (bis 1973 nur unbescholtenen) Mädchen, sanktionierte; der Tatbestand wurde erst 1997 abgeschafft.

16 Bis zum 1. StrRG vom 1.9.1969 war die einfache Homosexualität § 175 a. F., der Ehebruch $\S 172$ a. F. und die Sodomie $\S 175$ b a. F. strafbar gestellt; die Strafbarkeit der einverständlichen Homosexualität wurde erst endgültig 1997 abgeschafft (§ 175 sanktionierte vor 1969 jede Art von homosexuellem Verhalten, nach 1969 die homosexuellen Handlungen eines über 18jährigen an einem unter 18jährigen, ohne dass eine Schutzbefohleneneigenschaft vorliegen musste); der Tatbestand der Kuppelei $\S \S 180,181$ a. F. wurde 1973 zur „Förderung sexueller Handlungen Minderjähriger" in $\S 180$ StGB umgestaltet und mit dem sog. Erzieherprivileg ausgestattet (Abs. 1 S. 2). 
von 1973 nur marginale Änderungen erfahren hatte, so dass bis 1997 nur der erzwungene Beischlaf als Vergewaltigung galt, der Mann vom Opferkreis ausgeschlossen und die eheliche Vergewaltigung vom Tatbestand ausgenommen war. ${ }^{17}$ Die Verweigerung des Geschlechtsverkehrs durch die Ehefrau wurde sogar als Angriff durch Unterlassen auf das Rechtsgut der ehelichen Lebensgemeinschaft angesehen, so dass beispielsweise Horn diesbezüglich ein Notwehrrecht diskutierte. ${ }^{18}$ Die Einstufung der ehelichen Vergewaltigung als eine Art „Selbsthilfe“"19 bzw. auf Grund des in der Ehe bestehenden „Grundkonsenses“ zum regelmäßigen Geschlechtsverkehr als ,,weniger intensiv“ ${ }^{20}$ war und ist keine Seltenheit.

Hervorzuheben ist: Sämtliche Rollenzuschreibungen stellen sich auch als männerfeindlich dar. Im Bereich des Sexualstrafrechts führt diese Diskriminierung jedoch, wie zu zeigen sein wird, zu einer Privilegierung. ${ }^{21}$

\section{Das Rechtsgut des $\S 177$ StGB}

Bis 1973 war der 13. Abschnitt des StGB mit „Verbrechen und Vergehen wider die Sittlichkeit“ überschrieben. Als Rechtsgüter neben der Sittlichkeitsordnung und dem geordneten Sexualleben, waren in $\S 177$ StGB auch die sexuelle Freiheit und die Geschlechtsehre ${ }^{22}$ anerkannt. Das Reichsgericht betonte, ${ }^{23}$ dass durch die $\S \S 176 \mathrm{ff}$. StGB „vornehmlich“ der geschlechtlichen Freiheit und Integrität Schutz zuteil werde. Die Geschlechtsehre sollte dagegen primär in den Anwendungsbereich der $\S \S 185$ ff. StGB fallen. Das Wort „vornehmlich“ deutet an, dass die Geschlechtsehre als Rechtsgut weiterhin eine Rolle spielte, ${ }^{24}$ wodurch der Begriff der Bescholtenheit ${ }^{25}$ seinen Einfluss auf $\S 177$ StGB behielt. ${ }^{26}$ Dieser kann auf eine lange Tradition zurückblicken. Konnten ursprünglich überhaupt nur unbescholtene ${ }^{27}$ Frauen Opfer einer Vergewaltigungstat

17 Davor kam lediglich eine Bestrafung wegen $\S 240$ StGB und $\S 223$ StGB in Betracht; vertiefend Hanisch, Vergewaltigung in der Ehe, 1988.

18 Horn, ZRP 1985, 265 ff. zeigt durch seine Ausführungen ausgeprägtes Verständnis dafür, dass der den Verkehr verweigernden Ehefrau ein Angriff durch Unterlassen vorgeworfen wird, dessen sich der Ehemann durch Gewalt erwehren darf.

19 Schroeder, JZ 1999, 827 ff., 828.

20 Schünemann, GA 1996, 307 ff., 316.

21 Schon Jäger, Strafgesetzgebung und Rechtsgüterschutz bei Sittlichkeitsdelikten, 1957, S. 45, hält die „Zurückhaltung des Strafrechts gegenüber Gewaltakten in der Ehe“ für unverständlich: „Als Erklärung für diese Rechtssituation können nur die bürgerlichen Wertungen des vorigen Jahrhunderts gelten mit der für sie typischen Privilegierung des Mannes“.

22 Levy, Die Gewaltanwendung beim Sittlichkeitsdelikt, 1932, S. 34 ff.; v. Liszt, Lehrbuch des deutschen Strafrechts, 16. und 17. Auflage (1908), S. 360.

23 RGSt 24, 201 f., 202.

24 Vgl. u. a. BGH GA 1956, 317; BGHSt 11, 100, 102.

$25 \mathrm{Sch} / \mathrm{Sch}, 14$. Auflage (1969), § 182 Rn. 2: „Bescholten ist regelmäßig ein Mädchen, das vorher freiwillig und bewusst den Beischlaf gestattet hat; darüber hinaus begründet aber auch sonstiges in der sittenlosen Gesinnung des Mädchens wurzelndes unzüchtiges Treiben die Annahme geschlechtlicher Bescholtenheit"“.

26 Deutlich sichtbar am Institut des minder schweren Falls; dazu unten.

27 Die Frau musste entweder noch Jungfrau sein oder verheiratet und außerehelich unberührt; dazu Hundertmark, Streit 1986, S. 56. 
sein (Art. 119 CCC), wurde seit dem Allgemeinen Preußischen Landrecht von 1794, kurz ALR, auch die bescholtene Frau in den Tatbestand miteinbezogen. Allerdings war in $§ 1058$ ALR geregelt, dass die Strafe gemildert werden konnte, wenn die Person schon vorher in dem Ruf einer schlechten und liederlichen Lebensart gestanden hatte. ${ }^{28}$ Seit dem 4. StrRG, mit dem die Abkehr vom Moralstrafrecht fortgesetzt und bestätigt wurde, ${ }^{29}$ ist Rechtsgut des 13. Abschnitts das sexuelle Selbstbestimmungsrecht. ${ }^{30}$ Diese Veränderung der tatbestandlichen Schutzrichtung wurde allerdings nicht konsequent umgesetzt: Ansonsten hätte man zum einen die eheliche Vergewaltigung strafbar stellen, zum anderen die strikte Differenzierung zwischen $\S 177$ StGB und $\S 178$ StGB aufgeben sowie den Mann als vergewaltigungsfähig anerkennen müssen. Durch die Trennung von $\S 177$ StGB und $\S 178$ StGB, der sexuellen Nötigung, wurde weiterhin insistiert, dass die männliche außereheliche Penetration einer Frau im Vergleich zu sonstigen Penetrationen als schwereres Unrecht anzusehen sei, insbesondere auch wegen der damit verbundenen Gefahr einer unerwünschten Schwangerschaft. ${ }^{31}$

\section{Das zentrale Kernstück des Tatbestands: Das Nötigungsmittel der Gewalt}

\section{Unwiderstehliche Gewalt}

Die Nötigung kraft Gewalt ist in allen deutschgeschichtlich bedeutsamen Rechtsordnungen prägend für das Delikt der Notzucht. Ursprünglich war es das einzige tatbestandliche Nötigungsmittel. ${ }^{32}$ Erst im ALR, $§ 1051$, wurden ,gefährliche Bedrohungen des Lebens und der Gesundheit"als ebenfalls tattauglich anerkannt, allerdings mit einem milderen Strafrahmen versehen. ${ }^{33}$ Schon das ALR spricht traditionsgemäß in $\S 1052$ ALR von unwiderstehlicher Gewalt. Die Anforderung der Unwiderstehlichkeit fließt bis heute in die Auslegung des Gewaltbegriffs ein und verfestigte das traditionelle ${ }^{34}$ Bild von einer „Klassischen Vergewaltigung“ über die Jahrhunderte: Ein fremder Täter zwingt sein weibliches Opfer unter Anwendung erheblicher Gewalt unter Überwindung eines ernstlich geleisteten Widerstands zum außerehelichen Geschlechtsverkehr. ${ }^{35}$ In der Aufklärungszeit wurde vielfach und unter anderem von Voltaire bestritten, dass eine Notzucht trotz „ernstlichen Widerstreben des Weibes“ überhaupt verübt werden konnte. ${ }^{36}$ Noch 1928 bat der Abgeordnete Moses - ohne damit auf Widerspruch zu stoßen in Betracht zu ziehen, ,,dass es physisch außerordentlich schwer, ja fast unmöglich sei,

28 Vgl. v. Liszt/Schmidt, Lehrbuch des deutschen Strafrechts, 25. Auflage (1927), S. 544.

29 (Fn 16).

$30 \mathrm{Zu}$ den Kontroversen bezüglich dieses Begriffs Sick, Sexuelles Selbstbestimmungsrecht und Vergewaltigungsbegriff, 1993, S. $79 \mathrm{ff}$.

31 Ebenso Schapira, KJ, 221 ff., 230.

32 Vgl. Art 119 CCC.

33 Die Strafandrohung betrug bei Gewalt sechs bis acht Jahre Festungsstrafe, bei Drohung drei bis fünf Jahre.

34 U. a. Heffter, Lehrbuch des Gemeinen Deutschen Strafrechts, 6. Auflage (1857), S. 232, S. 233 Fn. 5.

35 Vgl. zuletzt BGH StV 2008, 81.

36 Dazu v. Liszt/Schmidt, Lehrbuch des deutschen Strafrechts, 25. Auflage (1927), S. 544; Henke, Handbuch des Criminalrechts und der Criminalpolitik, 1826, S. $202 \mathrm{ff}$. 
eine Frau mit Gewalt zum vollendeten Beischlaf zu bringen, solange ihre physische Widerstandskraft nicht gebrochen sei. In den Kliniken werde oft gezeigt, welche unge-

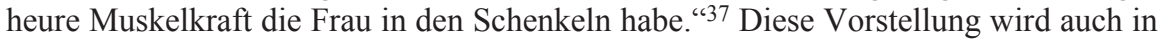
gerichtsmedizinischer Fachliteratur genährt. ${ }^{38}$ Versinnbildlicht werden diese Ansichten durch die Gleichnisse „vom Degen und der Scheide“ sowie „dem Nähfaden und dem Nadelöhr".39

\section{Die Rechtsfigur der vis haud ingrata ${ }^{40}$}

Der Begriff und die Vorstellung von einer vis haud ingrata sind schon in der Carolina gängig und finden sich u. a. im Lehrbuch des Gemeinen Deutschen Strafrechts von Wilhelm Heffter von 1857. ${ }^{41}$ Der Ausdruck bedeutet ,nicht unwillkommene Gewalt“. Diese soll dann vorliegen, wenn sich die Frau nur aus Scham gegen den Geschlechtsverkehr ziert. ${ }^{42}$ Das Sträuben der Frau ist in diesen Fällen nicht ernstlich, so dass der Mann auf gegenteilige Willensäußerungen keine Rücksicht mehr nehmen muss. Wann lediglich ein Zieren gegeben sein soll, erschließt sich „hauptsächlich aus der Art und dem Maß des geleisteten Widerstands“ und u. a. ,,aus der von dem Frauenzimmer selbst dargebotenen, aber nicht vermiedenen verführerischen Gelegenheit, aus dem unterlassenen oder schwachen Hilfsgeschrei, wo Hilfe möglich war" ${ }^{43}$ Insgesamt muss das Tatbild von der Gewalttätigkeit des Täters geprägt sein und nicht von der Nachgiebigkeit der Geschwächten. ${ }^{44}$ Konsequenz dieser Denkfigur: Sagt die Frau „nur“ „Nein“ oder wehrt sie sich nicht „ernstlich“, meint sie in Wirklichkeit „Ja“. Der BGH hat in einem Urteil von 1955 ausgeführt, dass in Konstellationen, in denen eine Frau dem Geschlechtsverkehr nur mit Worten widerspricht, ohne sich körperlich zu wehren, der Mann in der Regel ihr Einverständnis dazu als gegeben ansehen könne und auch eine Einwilligung in die eventuell gleichfalls damit verbundene Ehr- oder Körperverletzung annehmen dürfe. ${ }^{45}$ Bezeichnend ist, wie sich Hanack in seinem Gutachten zur Revision des Sexualstrafrechts, in dem er sich für die damalige Zeit sehr fortschrittlich für die

37 Verhandlungen des Strafrechtsausschusses des Reichstages über den Entwurf eines Allgemeinen Deutschen Strafgesetzbuches, BD. II, 21. Ausschuss, IV. Wahlperiode 1928, 81. Sitzung, Berlin 1932, S. 1 ff., S. 3 f.; auch Ort, Die Notzucht, 1932, S. 23 geht davon aus, dass die Frau die Vollendung verhindern. könne.

38 Möllhoff/Mueller, Gerichtliche Medizin, 2. Auflage (1975), S. 1100.

39 Dazu Henke, Handbuch des Criminalrechts und der Criminalpolitik, 1826, S. 202 ff.

40 U. a. Frank, Das StGB für das deutsche Reich, 1908, § 176 I; v. Liszt/Schmidt, Lehrbuch des deutschen Strafrechts, 25. Auflage (1927), § 105 S. 35; Schaeffer/Hinüber, Strafrecht Besonderer Teil, 1932, S. 40 Abs. 4 Ziff. 1 a; Mezger, Strafrecht Besonderer Teil II, 1949, $\S 24$ I; Maurach, Strafrecht BT I, 10. Auflage (2009), § 18 Rn. 17; Hanack, Zur Revision des Sexualstrafrechts in der BRD, 1968, Rz. 54.

41 Heffter, Lehrbuch des Gemeinen Deutschen Strafrechts, 6. Auflage (1857), Tit. 8, I § 293 Fn. 5.

42 U. a. BGHSt 22, 154 ff., 155.

43 Heffter, Lehrbuch des Gemeinen Deutschen Strafrechts, 6. Auflage (1857), Tit. 8, I § 293 Fn. 5.

44 Heffter, Lehrbuch des Gemeinen Deutschen Strafrechts, 6. Auflage (1857), Tit. 8, I § 293 Fn. 5.

45 BGH GA 1956, 317. 
Abkehr vom Moralstrafrecht einsetzte, äußert. Er kritisiert die Auffassung, dass an den „Irrtum des Täters über die Gewaltsamkeit des Beischlafs“" strenge Anforderungen zu stellen seien, ${ }^{46}$ da die Vergewaltigungssituation meistens durch die Frau in ,erheblicher Weise provoziert" worden und durch ein sexuelles Entgegenkommen von Seiten der Frau geprägt sei. ${ }^{47}$ Konsequenterweise müsse sich der Täter über das Vorhandensein eines Einverständnisses mit dem späteren Geschlechtsverkehr nicht mehr vergewissern. Vielmehr kehre sich die „Beweislast“ - zumindest aus Tätersicht - um. ${ }^{48}$ Die Rechtsfigur der vis haud ingrata führte dazu, dass der Vortrag des Täters, er sei von einem anfänglichen Zieren ausgegangen, als glaubhaft eingestuft und zugelassen wurde, selbst wenn die Tatsituation evident gegen ein bloßes Zieren sprach. ${ }^{49}$ Erinnert sei hier an die obigen Ausführungen bezüglich der geschlechtsspezifischen Rollenerwartungen. Auf der einen Seite wurde die Frau zur Passivität erzogen, auf der anderen Seite wurde von ihr in Vergewaltigungssituationen regelrecht verlangt, sich bis zum Äußersten zu verteidigen.

Mit dem Rechtsgrundsatz der vis haud ingrata hängt die Vorstellung vom natürlichen Masochismus der Frau zusammen. Auf Grund der Sexualentwicklung, den Menstruations- und Gebärschmerzen wird auf eine natürliche Passivität und einen weiblichen Masochismus geschlossen, der sich auch in der sexuellen Interaktion äußere. ${ }^{50}$ Dies führte zur Annahme der Rechtsprechung, dass eine Frau durch die Vornahme von Gewalt so erregt werden könne, dass sie schließlich in den Geschlechtsverkehr einwillige. ${ }^{51}$ Der BGH hielt den Täter in derartigen Konstellationen ${ }^{52}$ lediglich einer Nötigung zur Unzucht (§ 176 I Nr. 1 StGB a. F.) und nicht einer versuchten Notzucht für schuldig, da „sein Entschluss nicht auf die gewaltsame Vornahme des Beischlafs gerichtet“ gewesen sei. ${ }^{53}$

Auch der Verführungstatbestand, $\S 182$ StGB a. F., muss in diesem Kontext betrachtet werden. Die Verführung und die Vergewaltigung wurden nach gängiger Rechtspre-

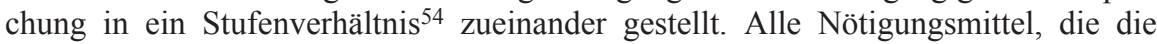
Schwelle des $\S 177$ StGB nicht erreichten, also die Drohung mit einem empfindlichen

46 Eine diesbezügliche gewissenhafte Prüfung wurde zumindest auf dem Papier vom BGH gefordert; dazu BGH, Urteil vom 14.7.1955 - 1 StR 728/54 m. w. N. Allerdings wird die Voraussetzung der ,gewissenhaften Prüfung “ nicht streng gehandhabt, sondern scheint nur dann eine Rolle zu spielen, wenn das Opfer sich nicht unerheblich gewehrt hat; vgl. BGH NJW $1968,1888$.

47 Hanack, Zur Revision des Sexualstrafrechts in der BRD, Rz. 55.

48 Hanack, Zur Revision des Sexualstrafrechts in der BRD, 1968, Rz. 55.

49 In BGHSt 22, $154 \mathrm{ff}$. hatte der Täter unter Vorhaltung einer Schreckschusspistole ein Mädchen zum Beischlaf gezwungen, das LG sah sich jedoch nicht imstande, dem Täter eine Drohung nachzuweisen.

50 Dazu Krafft-Ebing, Psychopathia sexualis, 1984; Deutsch, Psychologie der Frau, 4. Auflage (2000), S. 218 ff.; Freud, Drei Abhandlungen zur Sexualtheorie und verwandte Schriften, 1971; kritisch de Beauvoir, Das andere Geschlecht, 9. Auflage (2008), S. 486 ff.

51 BGH NJW 1965, $1284 \mathrm{f}$.

52 Vgl. auch BGH GA 1968, $84 \mathrm{f}$.

53 BGH NJW 1965, $1284 \mathrm{f}$.

54 BGHSt 22, 154 ff., 156 f.: „Die Bestimmung des $§ 177$ StGB betrifft also, soweit das Opfer ein unbescholtenes Mädchen unter 16 Jahren ist, eine Steigerung der Verführung.“; BGH NJW 1950, 710. 
Übel und die rein psychische Gewalt, wurden zum Tatbestand der Verführung gezählt, ${ }^{55}$ obwohl deren Einsatz den Charakter einer Verführung verfälscht. Das Opfer vollzieht den Geschlechtsverkehr mit dem „Partner“ in diesen Zwangssituationen ja gerade nicht einverständlich. Die Vergewaltigung erfuhr durch diese Wertung eine Herabstufung zu einer lediglich qualifizierten Verführung.

\section{Die Auslegung des Tatbestandsmerkmals der Gewalt durch die Rechtsprechung}

Die Rechtsprechung zum Nötigungsmittel der Gewalt in $\S 240$ StGB, der Nötigung, und $\S 177$ StGB verliefen im gleichen Zeitraum diametral.

Die Rechtsprechung und auch die Wissenschaft zu § 240 StGB gingen von der körperlichen Kraftentfaltung auf Täterseite immer mehr ab und legten ihren Schwerpunkt auf den körperlich wirkenden Zwang beim Opfer. ${ }^{56}$ Sogar psychisch vermittelter Zwang genügte zeitweilig. ${ }^{57}$ Das Rechtsgut des $§ 240$ StGB, die Willensentschließungs- und Willensbetätigungsfreiheit, ${ }^{58}$ erfuhr durch diese Vorgehensweise einen umfassenden Schutz. ${ }^{59}$

Im Rahmen des $\S 177$ StGB wurde das Tatbestandsmerkmal der Gewalt sehr restriktiv $^{60}$ ausgelegt. Unverzichtbar waren danach Gewalt gegen eine Person und eine ,- nicht notwendig erhebliche - körperliche Kraftentfaltung " und die Empfindung körperlichen und nicht nur seelischen Zwangs. ${ }^{61}$ Konsequenz waren Strafbarkeitslücken. ${ }^{62}$ Dabei waren die Konstellationen ${ }^{63}$ problematisch, in denen sich das Opfer auf Grund der Annahme, dass es sich in einer ausweglosen, hilflosen Lage befände, nicht mehr wehrte oder nicht erheblich genug, aber mit Worten oder konkludent seinen Nicht-Willen bekundete. Trotz empirischer Untersuchungen, die zeigten, dass das Opfer sich in ungefähr $50 \%$ der Fälle dem Täter ohne großen Widerstand beugt, ${ }^{64}$ insistierte man, dass das typische Opferverhalten erheblicher Widerstand sei. Man wollte nicht anerkennen, dass die Deliktssituation des $\S 177$ StGB oft die Lähmung der Motorik, die durch Zwangssituationen oder extreme Bedrohungssituationen hervorgerufen wird, nach sich zog. ${ }^{65}$

$55 \mathrm{Sch} / \mathrm{Sch}, 14$. Auflage (1969), § 182, Rn. 4, 9.

56 Vgl. dazu Sch/Sch-Eser, 27. Auflage (2006), Vorbem $\S \S 234$ ff. Rn. 7.

57 BGH NStZ 1981, 218 führt aus, dass psychische Einwirkungen ausreichen, wenn diese durch physische Kraftentfaltung verursacht wurden; insgesamt dazu Sch/Sch-Eser, 27. Auflage (2006), Vorbem $\S \S 234$ ff. Rn. 7.

58 Sch/Sch-Eser, 27. Auflage (2006), § 240 Rn. 1.

59 Ebenso Müller-Dietz, GA 1974, 33 ff., 48.

60 Dazu Laubenthal, Sexualstraftaten, 2000, S. 39 m. w. N. und die umfassende Analyse bei Sick, Sexuelles Selbstbestimmungsrecht und Vergewaltigungsbegriff, 1993, S. 96 ff.

61 U. a. BGH NStZ 1985, 71.

62 BT-Drs. 13/7324, S. 2; Sick, JR 1993, 164 ff., 166; Laubenthal, Sexualstraftaten, 2000, S. 40.

63 U. a. BGH NStE Nr. 26 zu § 177 StGB; BGH MDR 1981, 857; BGH NStZ 1990, 335; BGH NStZ 1995, 230; BGH NStZ-RR 1998, 104; BGH NStZ 1998, 106; eingehend dazu Sick, Sexuelles Selbstbestimmungsrecht und Vergewaltigungsbegriff, 1993, S. $100 \mathrm{ff}$.

64 Baurmann, Sexualität, Gewalt und psychische Folgen, 1996, S. 319; Rössner, FS Leferenz, 1983, 532.

65 Man spricht hier von einer ,tonischen Immobilität“, vgl. Hermann-Kolb, Die Systematik der sexuellen Gewalt- und Missbrauchsdelikte nach den Reformen 1997, 1998 und 2004, 2005, S. 17 Fn. 26. 
Dabei wurden regelmäßig Erwägungen hinsichtlich bestehender, aber nicht wahrgenommener Fluchtmöglichkeiten herangezogen, wenn es um die Frage nach der Ausübung von Gewalt ging. ${ }^{66}$ Eine körperliche Zwangswirkung wurde letzten Endes häufig verneint - selbst dann, wenn diese eindeutig gegeben war ${ }^{67}$ - eine psychische als nicht ausreichend angesehen. Auch das Vorliegen einer konkludenten Drohung wurde in diesen Konstellationen nur sehr selten bejaht, ${ }^{68}$ so dass der Täter letzten Endes nicht wegen $\S 177$ StGB verurteilt wurde. Und dies, obwohl sich die Annahme einer konkludenten Drohung mit Leib- oder Lebensgefahr in den meisten Konstellationen geradezu aufdrängte. $^{69}$

Die beschriebene restriktive Auslegung des Gewaltbegriffs konservierte die traditionellen Auslegungsgrundsätze und Deutungsmuster im Zusammenhang mit dem Vergewaltigungstatbestand. Sie überdehnte dabei regelmäßig die inhaltlichen Anforderungen an das Tatbestandsmerkmal der Gewalt und wurde insbesondere der spezifischen Deliktssituation des $\S 177$ StGB nicht gerecht. Der Charakter der Gewalt musste auch nach damaliger Auffassung unstrittig nicht unwiderstehlich sein. Es war vor allem nicht relevant, ob die Genötigte dem Zwang durch Widerstand, Flucht oder Anrufung fremder Hilfe hätte entgehen können. ${ }^{70}$ Derartige Erwägungen als einschränkendes Korrektiv auf Tatbestandsebene waren infolgedessen verfehlt. Die Rechtsprechung übersah eine notwendig vorzunehmende Differenzierung: Nicht der Beischlaf muss mittels Gewalt vollzogen werden, sondern ausweislich des $§ 177$ I StGB der Wille der Genötigten mit Gewalt gebrochen werden, um sodann den Geschlechtsverkehr gegen deren Willen durchführen zu können. Schließlich stellt die vis compulsiva neben der vis absoluta eine anerkannte Gewaltform in $\S 177$ StGB dar. Die ,viktimologische Restriktion“ des Gewalt- bzw. Drohungsbegriffs auf Tatbestandsebene stand des Weiteren im Widerspruch zur ständigen Auslegung des Tatbestandsmerkmals „Irrtum“ in $\S 263$ StGB durch Rechtsprechung und h. M. in der Literatur, die dort eine derartige Restriktion ablehnen. ${ }^{71}$ Sogar „leichtsinnigen Opfern“ wurde und wird der strafrechtliche Schutz nicht versagt. Dazu der BGH: ${ }^{72}$ „Die viktimologisch motivierten Ansätze zur Einschränkung des Betrugstatbestands wegen geringerer Schutzbedürftigkeit des zweifelnden Tatop-

66 U. a. BGH StV 1981, $543 \mathrm{ff}$.

67 BGH, Beschluss vom 18.1.1995 - 3 StR 559/94: „Der Angeklagte nützte die Situation aus und...stürzte sich auf Katja, so daß er auf ihr zum Liegen kam. Sie weinte und empfand große Angst. In dieser Situation drückte er sein Geschlechtsteil an ihre Scheide, beließ es über mehrere Minuten dort und bewegte sich so auf ihr, daß sie Schmerzen im Scheidenbereich empfand.“ Der BGH verneinte das Vorliegen von § 178 a. F., „,da es nicht festgestellt sei, dass der Angeklagte bewusst Gewalt zur Erzwingung der sexuellen Handlung eingesetzt habe. Auch unter Berücksichtigung der Überlegenheit eines erwachsenen Mannes gegenüber einem Kind sei es denkbar, dass das Mädchen lediglich überrumpelt worden sei und nur subjektiv Angst empfunden habe.“

68 Hierzu exemplarisch BGH NStE Nr. 26 zu $§ 177$ StGB.

69 Ebenso MüKo-Renzikowski, 2005, §177 Rn. 48; Mildenberger, Schutzlos-HilflosWiderstandsunfähig, 1998, S. $39 \mathrm{ff}$.

$70 \mathrm{Sch} / \mathrm{Sch}, 14$. Auflage (1969), Vorbem $\S 234 \mathrm{ff}$. Rn. 14; Sch/Sch-Eser, 21. Auflage (1982), Vorbem $\S \S 234$ ff. Rn. 24; Sch/Sch-Eser, 27. Auflage (2006), Vorbem $\S 234$ ff. Rn. 24.

71 Küper, Strafrecht Besonderer Teil, 2008, S. 229; kritisiert u. a. von Schünemann, in Schünemann/Dubber, Die Stellung des Opfers im Strafrechtssystem, 2000, S. 1 ff., S. 5.

72 BGH NJW 2003, $1198 \mathrm{ff}$. 
fers finden im Wortlaut des $\S 263$ StGB keine Stütze ...Die ihr zu Grunde liegende Vorstellung, dass sich das Tatopfer bei solchen Zweifeln vergewissern oder von der schädigenden Vermögensverfügung Abstand nehmen könne, läuft auf eine dem Strafrecht fremde Bewertung eines Mitverschuldens hinaus, das auch sonst nicht tatbestandsausschließend wirkt, ..."

\section{Der minder schwere Fall-Plattform antiquierter Schuldzuschreibungen ${ }^{73}$}

Die Analysen der minder schweren Fälle ergeben, dass die Rechtsprechung die Vergewaltigung vornehmlich als ein Trieb- und Spontandelikt bewertete. ${ }^{74}$ Dass bei einer Vergewaltigung für den Täter die Ausübung von Macht und Dominanz sowie die Erniedrigung des Opfers im Vordergrund stehen, ${ }^{75}$ wurde verkannt.

\section{Der minder schwere Fall wegen Mitverschuldens}

Hierbei wird der Frau vorgeworfen, die Tat provoziert zu haben, „wenn auch ungewollt". ${ }^{\prime 6}$ Das Mitverschulden wurde dabei in sozial adäquaten Verhaltensweisen wie

73 Ein Drittel aller Verurteilungen zu $§ 177$ StGB a. F. wurden als „minder schwer Fall“ eingestuft, so dass von einem Ausnahmestrafrahmen nicht mehr die Rede sein kann; vgl. Albrecht, Strafzumessung bei schwerer Kriminalität, 1994, S. 293.

74 U. a. BGH MDR 1963, 62; BGH NStZ 1981, 222; BGH StV 1986, 149 f.; BGH-Urteil vom 25.4.1991, 4 StR 110/91; BGH-Beschluss vom 1.9.1993, 4 StR 495/93; Schneider, FS Lenckner, $1998,847$.

75 Vgl. Hauch, Gelebte Sexualität im Maßregelvollzug?, 2006, 18 ff., 19; Schneider, FS Lenckner, $1998,847$.

76 U. a. BGH MDR 1963, 62: „Auf jeden Fall hat das Mädchen, trotz seiner ständig gegenteiligen Erklärungen doch, wenn auch vielleicht unbewusst mit dem Feuer gespielt. Dies alles konnte das letzte brutale Vorgehen des jungen Mannes, so verwerflich es war, doch menschlich irgendwie verständlich erscheinen lassen.“; BGH NStZ 1981, 222; BGH, NStZ 1982, 26: „,.. der Umstand, dass der Angeklagte seine frühere Freundin bei der ersten Tat körperlich mißhandelt, sie gegen erkennbaren Widerstand ausgezogen, angeherrscht und bedroht hat, schließt nicht aus, daß er, "in weitaus stärkerem Umfang mit eigenen moralischen Hemmungen konfrontiert worden" wäre, "wenn U mehr körperlichen Widerstand gezeigt und geschrien hätte". Dies gilt auch dann, wenn dem sensiblen und zarten und eingeschüchterten Mädchen ein stärkerer körperlicher Widerstand oder wenigstens laute Hilferufe nicht möglich gewesen wären“; BGH StV 1986, 149 f.; BGH-Urteil vom 25.4.1991, 4 StR 110/91; BGH-Beschluss vom 1.9.1993, 4 StR 495/93: „So berücksichtigt (das $L G$ ) insbesondere nicht, daß die Verfehlung des Angeklagten als Spontantat zu werten ist, die sich aus einer - zumindest aus der Sicht des Angeklagten - erotisch gefärbten Beziehung zwischen Täter und Opfer zur Tatzeit ergeben hat. Zudem hat die später geschädigte Bettina P. - wenn auch unbeabsichtigt - mit ihrem Verhalten nicht unerheblich zu einer Fehleinschätzung der Situation durch den Angeklagten beigetragen. So hatte sie zunächst den Angeklagten in einer Gaststätte getroffen, um gemeinsam mit ihm den Abend zu verbringen. Auf ihre Veranlassung suchten beide später die Wohnung des Angeklagten auf, um dort Musik zu hören. Nachdem Bettina P. Schuhe und Jacke abgelegt hatte, begann sie nach der Musik zu tanzen. Auch wenn sich die Geschädigte im Hinblick auf ihre langjährige lose Bekanntschaft mit dem Angeklagten, in der es bislang zu keinen sexuellen Kontakten gekommen war, hierbei völlig arglos verhielt, so ist doch nachvollziehbar, daß der Angeklagte sich durch das Verhalten der Frau stimuliert fühlte und 
der bloßen Unterhaltung oder dem Begleiten eines Mannes in seine Wohnung gesehen. ${ }^{77}$ Durch die Anerkennung des Mitverschuldens wurde der Prozess dazu genutzt, das sexuelle Vorleben des Opfers zu sezieren und auf angeblich unmoralische und für die konkrete Tat irrelevante Aspekte hin zu untersuchen. Die Rechtsprechung war äußerst kreativ, wenn es um eine Entschuldigung für das Verhalten des Täters ging, wie u. a. das folgende Urteil des BGH vom 24.1.1990 zeigt. ${ }^{78}$ Die Geschädigte verdiente sich als Aktmodell Geld. Der Täter sollte diese zu einem Kunden fahren. Er suchte jedoch mit seinem Opfer einen Waldparkplatz auf. Mit einem Fahrtenmesser zwang er sie dazu, geschlechtlich mit ihm zu verkehren, danach Mundverkehr mit ihm auszuüben und sich schließlich in unbekleidetem Zustand von ihm fotografieren zu lassen. Die Anwendung des Ausnahmestrafrahmens war hier nach Ansicht des Gerichts geboten, da der sexuellen Tatprovokation durch das Opfer eine maßgebliche Rolle zugesprochen wurde. Diese wurde in dem Umstand gesehen, dass das Opfer Aktmodell steht. Dadurch wurde die „Hemmschwelle des Täters im sexuellen Bereich herabgesetzt“ ${ }^{\text {" }}{ }^{79}$ Hervorzuheben ist, dass die Tat ausweislich der Urteilsfeststellungen bereits Tage vorher geplant war.

\section{Sonstige Strafmilderungsgründe}

Als weiterer Strafmilderungsgrund war der des sexuellen Notstands bis in die neunziger Jahre explizit anerkannt. ${ }^{80}$ Strafmildernd wirkte sich dabei regelmäßig der Umstand aus, dass der Täter nicht verheiratet war und infolgedessen auch nicht regelmäßig durch Geschlechtsverkehr seine sexuelle Befriedigung finden konnte.

Auch die Tatsache, dass das Opfer eine Prostituierte war, wurde strafmildernd berücksichtigt. ${ }^{81}$

\section{Zusammenfassende Bewertung}

Die Vorstellung, dass eine Vergewaltigung nur unter erheblicher Gewaltanwendung möglich sei, ist im Vergewaltigungstatbestand seit Jahrhunderten fest verankert. ${ }^{82} \mathrm{Ge}$ walt wurde im Rahmen des $\S 177$ StGB immer in seinem ursprünglichsten Sinne verstanden. Je weniger sich die Gewalt als eine absolute darstellte, desto höher waren die Darlegungsanforderungen beim Opfer, dass es sich ausreichend gewehrt habe, da - ein-

sich Hoffnung auf die Aufnahme intimer Kontakte machte. Zwar vermag dies nicht zu entschuldigen, daß er den Geschlechtsverkehr mit der Geschädigten erzwang, nachdem er nach erstem Körperkontakt den entgegenstehenden Willen der Frau erkannt hatte, läßt seine Tat jedoch in milderem Licht erscheinen."

77 BGH StV 1986, $149 \mathrm{ff}$.

78 BGH NStE 1991 Nr. 23 zu § 177 StGB.

79 BGH NStE 1991 Nr. 23 zu § 177 StGB.

80 BGH-Urteil, 8.10.1971, 4 StR 517/79; BGH-Beschluss, 30.12.1980, 3 StR 490/80; BGHUrteil, 8.8.1984, 3 StR 256/84; Sch/Sch-Lenckner, 25. Auflage (1997), § 177 Rn. 17.

81 BGH-Beschluss, 18.4.1973, 4 StR 135/73; BGH StV 1995, 635; BGH StV 1996, 26 f.; dagegen BGH, 29.6.1971, 5 StR 235/71.

82 Dazu Weis, Die Vergewaltigung und ihr Opfer, 1982, S. 53. 
zigartig für das StGB - das Vorliegen von Gewalt anhand der Widerstandshandlungen des Opfers bestimmt wurde. Unter anderem das Institut der vis haud ingrata versuchte dies zu legitimieren. Die Vorstellung des Täters, selbst wenn diese evident von der wahrgenommenen Realität abwich, wurde stets berücksichtigt. ${ }^{83}$ Das Verhalten der Frau als Opfer wurde - im Unterschied zu anderen Willensbeugungs- und Interaktionsdelikten des $\mathrm{StGB}^{84}$ - stets dahingehend hinterfragt, ob diese nicht die Verletzung ihres sexuellen Selbstbestimmungsrechts mitzuverantworten hatte. Dabei wurde die Gefahr einer Vergewaltigung als ein typischer Kausalverlauf dargestellt, als Alltag der Frauen, die sich in Kontakt mit Männern dieses Umstands stets bewusst sein sollten. Die Widerstandshandlungen des Opfers wurden als Indikator für das Vorliegen von Gewalt i. S. d. $§ 177$ StGB behandelt, als nicht ausreichend bewerteter Widerstand als unterlassene Möglichkeit der Gewaltdeeskalation kritisiert. Die „Verantwortungsabgabe“ wird damit zu einem zentralen Begriff der Geschichte des $\S 177$ StGB. Dies zeigt sich nicht zuletzt an der Rechtsprechung zum minder schweren Fall. Hier leben die Mythen von der Frau als Verführerin und dem Mann, der diesem Verführungsakt willenlos ausgeliefert ist, auf. Wird das Urteil der sog. Bescholtenheit über das weibliche Opfer gesprochen, werden die Rollen des Tatgeschehens vertauscht. Das weibliche Agieren wird in den Vordergrund gerückt, das Opfer wird zum Urheber der Tat. Als Argument bzw. Erklärungsversuch für diese Vorgehensweise wurde regelmäßig die nach der alten Fassung drohende Freiheitsstrafe von nicht unter zwei Jahren angeführt. Dies kann jedoch nicht entschuldigen, dass Begründungsinhalte von außerrechtlichen und irrelevanten Erwägungen getragen werden.

\section{Das 33. St ̈̈G - ein Wendepunkt?}

Das 33. St $\ddot{A} G^{85}$ und das 6. StrRG ${ }^{86}$ brachten grundlegende Änderungen hinsichtlich der $\S \S 177$ StGB a. F., $178 \mathrm{StGB}$ a. F. mit sich, wobei hier vor allem das 33. StÄG von Interesse ist. ${ }^{87}$ Das 6. StrRG führte bezüglich $\S 177$ StGB hauptsächlich zu einer Harmonisierung der Strafrahmen mit denen der Raubdelikte. ${ }^{88}$

\section{Ein neues Nötigungsmittel, § 177 I Nr. 3 StGB}

Durch die Einführung von $\S 177$ I Nr. 3 StGB wurde ein neues Nötigungsmittel, die Ausnutzung einer schutzlosen Lage, geschaffen, das die oben gerügten Strafbarkeits-

83 BGHSt 22, 154 ff.; BGH-Urteil vom 25.4.1991, 4 StR 110/91: „Daß dem Angeklagten das fehlende Einverständnis von Frau M. bewußt war, besagt noch nichts darüber, ob er deren Widerstand auch mit Gewalt brechen wollte und ob er zu diesem Zweck seine Körperkräfte bewußt einsetzte oder ob es ihm in diesem Augenblick nicht nur um - wie er es in seiner richterlichen Vernehmung vom 21. März 1989 (UA 47) bekundete - die gewaltlose Erreichung des "Höhepunkts" ging, nachdem er "schon ganz kurz davor" war."

$84 \S \S 263,249,253,255$ StGB.

85 BGBl. I 1607.

86 BGBl. I 164.

87 Es ist nicht meine Intention, eine umfassende Bewertung der Reformen vorzunehmen.

88 Sch/Sch-Lenckner/Perron/Eisele, 27. Auflage (2006), Vorbem $\S § 174$ ff. Rn. 6. 
lücken teilweise schließen konnte. Auf der einen Seite wurde der Schutz Behinderter vor sexuellen Übergriffen erweitert, ${ }^{89}$ auch wenn dies zur problematischen Abgrenzungsfrage von $\S 179$ StGB führte. ${ }^{90}$ Auf der anderen Seite wurde dadurch gebilligt, dass es zahlreiche Opfer gibt, die „,vor Schrecken starr oder aus Angst vor der Anwendung von Gewalt durch den Täter dessen sexuelle Handlungen über sich ergehen lassen“91 und sich nicht wehren, diese Situation aber trotzdem als Vergewaltigung zu werten ist. Eine schutzlose Lage liegt vor, ,wenn die Schutz- und Verteidigungsmöglichkeiten des Opfers in einem solchen Maße verringert sind, dass es dem ungehemmten Einfluss des Täters preisgegeben ist. Dies ist regelmäßig der Fall, wenn das Opfer sich dem überlegenen Täter allein gegenüber sieht und auf fremde Hilfe nicht rechnen kann, wobei es allerdings eines gänzlichen Beseitigens jeglicher Verteidigungsmöglichkeiten nicht bedarf."92 Es genügt, wenn das Opfer auf Grund äußerer Umstände in einer für einen objektiven Beobachter nachvollziehbaren Weise Widerstand für aussichtslos und Hilfe Dritter für unerreichbar hält. ${ }^{93}$ Der Täter muss unter Ausnutzung der hilflosen Lage das Opfer zur sexuellen Handlung nötigen. Dabei wird die Frage, ob eine eigenständige Nötigungshandlung i. S. d. § 240 I StGB zusätzlich erforderlich ist, heftig diskutiert. Die überwiegende Rechtsprechung ${ }^{94}$ verneint diese Frage. ${ }^{95}$ Der Nötigungsbegriff ist hier nämlich in dem Sinne zu verstehen, dass der Täter sich die schutzlose Lage des Opfers bewusst zu nutze macht, um sexuelle Handlungen gegen den Willen des Opfers durchzuführen. Die Willensbeugung (also Nötigung) erfolgt hier mittels des Missbrauchs der Hilflosigkeit des Opfers, das sich aus der Angst vor Beeinträchtigungen für Leib oder Leben durch den Täter ergibt, weil dieser beispielsweise eine Vereinzelung des Opfers herbeigeführt hat. Wer die hilflose Lage geschaffen hat und worauf sie beruht, ist jedoch letztendlich irrelevant. ${ }^{96}$ Der Wille des Opfers muss aber aus Angst vor Gefahren für Leib oder Leben gebeugt werden und gerade diese Angst muss zur Duldung des sexuellen Ansinnens des Täters führen. ${ }^{97}$

Der Tatbestand der Vergewaltigung ist damit weiterhin nur dann erfüllt, wenn die körperliche Integrität betroffen ist. Psychische Gewalt wird nicht als vergewaltigungstaugliches Nötigungsmittel anerkannt.

89 Denn $§ 179$ StGB ist nur einschlägig, wenn absolute Widerstandsunfähigkeit gegeben ist.

90 Dazu u. a. Renzikowski, NStZ 1999, 377 ff., 379; Oberlies, ZStW 2002, $131 \mathrm{ff}$.

91 BT-Drs. 13/7324, S. 6.

92 In BGH NStZ 2000, 140 beschäftigte sich der 2. Strafsenat zum ersten Mal mit dem Anwendungsbereich des $\S 177$ I Nr. 3 StGB.

93 Sch/Sch-Lenckner/Perron/Eisele, 27. Auflage (2006), § 177 Rn. 9.

94 BGH NJW 1999, 369; BGH NJW 2000, 1048; BGH NStZ 2002, 199; NStZ-RR 2003, 42; zustimmend Laubenthal, Sexualstraftaten, 2000, Rn. 146.

95 Diese Auslegung wurde auch vom BVerfG NJW 2004, 3768 ff. für verfassungsgemäß erklärt; dagegen Sch/Sch-Lenckner/Perron/Eisele, 27. Auflage (2006), § 177 Rn. 8 ff.; Fischer, 57. Auflage (2010), § 177 Rn. 35.

96 Sch/Sch-Lenckner/Perron/Eisele, 27. Auflage (2006), § 177 Rn. 9.

97 BGH NStZ 2009, 263. 


\section{Ausgestaltung der Vergewaltigung als Regelbeispiel}

Die Ausgestaltung der Vergewaltigung als Regelbeispiel in $§ 177$ II Nr. 1 StGB wurde - wie die Zusammenfassung von sexueller Nötigung und Vergewaltigung in einem Tatbestand $^{98}$ - von der Intention flexibler Handhabung ${ }^{99}$ getragen, führte jedoch zu Kontroversen. ${ }^{100}$ Dabei stellt sich $u$. a. die Problematik des Versuchs einer Vergewaltigung, wobei bei vollendeter sexueller Nötigung nach § 177 I StGB ein Versuch des § 177 II Nr. 1 StGB ausgeschlossen sein soll. ${ }^{101}$ Die Ausgestaltung der Vergewaltigung als Regelbeispiel muss sich den Vorwurf der Unsystematik gefallen lassen, da diese im Rahmen der $\S \S 176$ a II Nr. 1 StGB und 179 V Nr. 1 StGB als Qualifikation geregelt wurde. Die Regelbeispielstechnik bringt auch keine sichtbaren Vorteile, da das Merkmal der besonderen Erniedrigung schon für sich zu der gewünschten Flexibilität führen würde. Die Zugeständnisse hinsichtlich des Verlustes der Verbrechensqualifikation, ${ }^{102}$ die in der gesonderten Erwähnung des Begriffs der Vergewaltigung im Titel des Tatbestands sowie in der Ausnahme von der Regel, dass der Begriff im Tenor aufzunehmen ist, ${ }^{103}$ zu sehen sind, können die Nachteile im Zusammenhang mit der Regelbeispielslösung nicht kompensieren.

Hervorzuheben ist, dass vom Begriff der Vergewaltigung nun nicht mehr nur vaginale Penetrationen erfasst werden, sondern auch anale und orale sowie solche, die mit anderen Gegenständen ${ }^{104}$ durchgeführt werden. ${ }^{105}$ Die notwendige Ausdehnung des Vergewaltigungsbegriffs ${ }^{106}$ wurde im Gegensatz zu $§ 176$ II Nr. 1 StGB aber mit der zusätzlichen Anforderung einer besonderen Erniedrigung in $\S 177$ II Nr. 1 StGB ausgestattet, was zu Unsicherheiten in der Rechtsanwendung führt und streitig diskutiert wird. ${ }^{107}$ Dabei geht es um die Frage, ob dem Merkmal der besonderen Erniedrigung bei sexuellen Handlungen, die mit einem Eindringen verbunden sind, überhaupt eine eigenständige Bedeutung zukommt. ${ }^{108}$ Zahlreiche Entscheidungen des BGH verlangen

98 MüKo-Renzikowski, 2005, § 177 Rn. 13.

99 MüKo-Renzikowski, 2005, § 177 Rn. 13, äußert die Ansicht, dass diese Umstufung das Ergebnis eines Kompromisses im Zuge der Einführung der ehelichen Vergewaltigung gewesen sei.

100 Renzikowski, NStZ 1999, 377, bezeichnet diese Ausgestaltung als Herabstufung; ähnlich Schroeder, JZ 1999, 827 ff., 829: er kritisiert, dass ,ein jahrtausendalter Tatbestand“ abgeschafft und zu einem „bloßen“ Regelbeispiel gemacht worden sei, womit der Verlust als „eigenständiger Verbrechenstyp“ einhergehe; kritisch auch Lenckner, NJW 1997, 2801 ff., 2802.

101 BGH NJW 98, 2987; BGH NStZ 03, 602; Sch/Sch-Lenckner/Perron/Eisele, 27. Auflage (2006), § 177 Rn. 18; ein Versuch des Grundtatbestands aus Abs. 1 und der Vergewaltigung aus Abs. 2 Nr. 1 ist nicht möglich.

102 Ebenso Lenckner, NJW 1997, 2802; Schroeder, JZ 1999, 829.

103 MüKo-Renzikowski, 2005, § 177 Rn. 118.

104 Dazu BGHSt 46, $225 \mathrm{ff}$.

105 MüKo-Renzikowski, 2005, § 177 Rn. 62 ff.

106 Gesetzgeberisches Motiv war es, auch das Eindringen mit Gegenständen zu erfassen, da dieses eine „,in gleicher Weise belastende und erniedrigende Verhaltensweise darstelle“, vgl. BT-Drs. 13/2463, S. 7.

107 Sch/Sch-Lenckner/Perron/Eisele, 27. Auflage (2006), § 177 Rn. 20; MüKo-Renzikowski, 2005, § 177 Rn. 65 m. w. N.

108 Dagegen MüKo-Renzikowski, 2005, § 177 Rn. 65. 
eine wertende Betrachtung der Umstände des Einzelfalls, ob das Opfer „,besonders erniedrigt" wurde. ${ }^{109}$ Bei Beischlaf, Anal- und Oralverkehr soll die besondere Erniedrigung regelmäßig angenommen werden. ${ }^{110}$ Dieses Merkmal ist jedoch im vorurteilsbehafteten Tatbestand des $\S 177$ StGB problematisch, da es als Einfallstor für Erwägungen genutzt wird, die an das Merkmal der „Bescholtenheit“ erinnern, wenn etwa der 4. Strafsenat ${ }^{111}$ ausführt: „Grundsätzlich bedarf es aber jeweils der positiven Feststellung der Umstände des Einzelfalls, die in wertender Betrachtung die Annahme der „besonderen Erniedrigung“ des Tatopfers stützen. Daran fehlt es hier. Die Feststellungen lassen die Möglichkeit offen, dass die vom Angeklagten erzwungenen sexuellen Handlungen einschließlich des Oralverkehrs ihrer Art nach von der getroffenen Verabredung zum entgeltlichen Sexualverkehr umfasst waren. Deshalb ist nach ständiger Rechtsprechung des Senats die grundsätzliche Bereitschaft des Tatopfers zu sexuellen Handlungen regelmäßig ein für die Beurteilung des Schuldgehalts der nach $\S 177$ StGB qualifizierten Tat bestimmender Umstand.“

\section{Erweiterung des persönlichen Anwendungsbereichs}

Die eheliche Vergewaltigung wurde nach heftigen Kontroversen ${ }^{112}$ durch die Streichung des Wortes ,außerehelich“ im Tatbestand des $§ 177$ StGB strafbar gestellt, wobei dies auch ablehnende Stimmen nach sich zog. ${ }^{113}$ Der Mann wurde in die Neufassung des $\S 177$ StGB als Vergewaltigungsopfer miteinbezogen.

109 BGH NStZ 2000, 255; BGH NJW 2000, 672; BGH NStZ 2001, 598; nach u. a. BGH NStZ 2001, 598 und BGH NStZ 2004, 440 wird das Opfer in der Regel auch durch das Einführen eines Fingers in Scheide oder After besonders erniedrigt.

110 MüKo-Renzikowski, 2005, § 177 Rn. 65, er kritisiert, dass die besondere Erniedrigung damit zu einer Ausgrenzung bestimmter Eindringensformen führe.

111 BGH NStZ 2001, 369: ,a) Nach den rechtsfehlerfrei getroffenen Feststellungen hatte der Angekl. mit der Geschädigten, die als Prostituierte tätig war, für den Abend des Tattags einen „Hausbesuch“ in seiner Wohnung für die Dauer von zwei Stunden und einen vereinbarten Preis von 500 DM verabredet. Da er jedoch über kein Geld verfügte, um sie „für ihre Dienste zu bezahlen“, hatte er von vornherein den Entschluss gefasst, mit ihr ,auch gegen ihren Willen sexuelle Handlungen durchzuführen“. Nachdem Frau H erschienen war, verschloss er die Wohnungstür. Bald darauf zog er ein Messer und eine Wäscheleine bzw. einen Strick hervor und forderte Frau $\mathrm{H}$,im Befehlston auf, sich auszuziehen, wobei er ihr das Messer entgegenhielt", was sie aus Angst tat. Nachdem er sich ebenfalls entkleidet hatte, musste sie sich auf den Bauch legen. Sodann legte er sich auf sie und führte „geschlechtsverkehrsähnliche Bewegungen“ aus, wobei er das Messer „in Reichweite“ ablegte. Anschließend musste die Geschädigte mit ihm den Oralverkehr ausüben. „Dabei hielt er das Messer wieder in der Hand". Schließlich legte er sich wieder auf sie, steckte sein Glied zwischen ihre Brüste und gelangte so zum Samenerguss“; Lenckner, NJW 1997, 2802, hat genau diese Vorgehensweise der Rechtsprechung befürchtet.

112 Letztendlich wurde die eheliche Vergewaltigung weder als ein Antragsdelikt ausgestaltet noch mit einer Versöhnungs- Vollstreckungsklausel oder Widerspruchsklausel versehen; dazu Wetzel, Die Neuregelung der $\S$ 177-179 StGB unter besonderer Berücksichtigung des ehelichen Bereichs und ausländischer Rechtsordnungen, 1998, S. $66 \mathrm{ff}$.

113 Vgl. Schroeder, JZ 1999, 827 ff., 828; Schünemann, GA 1996, 307 ff., 316. 


\section{Der minder schwere Fall einer Vergewaltigung, § 177 I StGB und $\S 177 \mathrm{~V} 1$. Hs. StGB}

Dogmatisch gesehen ist es inkorrekt, von einem minder schweren Fall einer Vergewaltigung zu sprechen, da die Vergewaltigung nun ein Regelbeispiel darstellt und sich Abs. 5 Hs. 1 auf Abs. 1, die sexuelle Nötigung bezieht. Im Zuge der Reformdiskussion wurde teilweise auch vertreten, dass es auf Grund der Systematik des neuen $\S 177$ StGB keinen minder schweren Fall mehr geben könne. ${ }^{114}$ Es wurde aber auch befürchtet, dass eheliche Vergewaltigungen generell als minder schwer eingestuft werden würden. ${ }^{115}$ Die Gesetzesmaterialien zum 33. StÄG ${ }^{116}$ und dem 6. StrRG ${ }^{117}$ sprechen sich nicht für eine Abschaffung des minder schweren Falls der Vergewaltigung aus. Schroeder erhoffte sich aber, dass „die zu häufige Annahme minder schwerer Fälle reduziert"118 werde, da der Richter einem höheren Begründungszwang ausgesetzt sei. Die damit zusammenhängende Vermutung, ${ }^{119}$ dass die Gerichte bei Verneinung der Indizwirkung des Regelbeispiels in $\S 177$ II Nr. 1 StGB stets auf den Grundtatbestand des $\S 177$ I StGB zurückgreifen würden, hat sich nicht bewahrheitet. Der BGH hat inzwischen in zahlreichen Konstellationen „den minder schweren Fall einer Vergewaltigung“ über Abs. 5 in Betracht gezogen. ${ }^{120}$

\section{Vorhergehende intime Beziehungen ${ }^{121}$}

Bestanden zwischen Täter und Opfer vor der Tat intime Beziehungen, wobei egal ist, ob diese im Rahmen einer Ehe oder einer sonstigen Partnerschaft gepflegt wurden, so ist dies nach der Rechtsprechung stets ein Indiz für das Vorliegen eines minder schweren Falls. ${ }^{122}$ Teilweise wird dabei für erheblich gehalten, ob der Täter für die Beziehung

114 Frommel, Sachverständigenanhörung des Rechtsausschusses vom 06.12 1995, S. 2; Frommel, KJ 1996, 164 ff., 169; Desseker, NStZ 1998, 1 ff., 2.

115 Frommel, KJ 1996, 164 ff., 169.

116 Vgl. BT-Drs. 13/7324, S. 5 ff.

117 Vgl. BT-Drs. 13/9064, S. 12 f.

118 Schroeder, Zusammenstellung der Stellungnahmen zur Anhörung des Rechtsausschusses vom 7.12.1995, S. 66; in JZ 1999, 829 vermutet ders. in der Umgestaltung der Vergewaltigung zu einem Regelbeispiel einen „Trick“ des Gesetzgebers, die Regel der Annahme von minder schweren Fällen durch die Rechtsprechung zu durchbrechen.

119 Wetzel, Die Neuregelung der $\S \S 177-179$ StGB unter besonderer Berücksichtigung des ehelichen Bereichs und ausländischer Rechtsordnungen, 1998, S. 191.

120 BGH NStZ 2000, 419; BGH NStZ 2001, 366 f.; BGH NStZ 2003, 202; BGH NStZ 2004, 32; BGH StV 2006, 523 f.; BGH NStZ-RR 2006, 6 f.; BGH StraFo 2007, 472; BGH StV $2008,81$.

121 BGH StV 1998, 76; BGH StV 2000, 557 ff.; BGH StV 2001, 453; BGH NStZ-RR 2002, 9, wobei der BGH es dem Täter zugute halten will, dass er eine niedrigere Hemmschwelle zu überwinden hatte, da Täter und Opfer aus einem Kulturkreis stammen, in welchem von der Ehefrau Unterordnung und Gehorsam erwartet wird; BGH NStZ-RR 2003, 168; BGH StraFo 2007, 472; BGH NStZ-RR 2006, 6 f.

122 MüKo-Renzikowski, 2005, § 177 Rn. 108; Sch/Sch-Lenckner/Perron/Eisele, § 177 StGB Rn. 33 bezeichnet diese Vorgehensweise richtigerweise als ,recht weitgehend“; BGH NStZ 2000, 254 betrifft den Fall, in dem das LG einen minder schweren Fall nach § 177 V 
ungewöhnliche Sexualpraktiken erzwungen hat. ${ }^{123}$ Der 4. Strafsenat nimmt selbst dann einen minder schweren Fall an, wenn der Täter erhebliche Gewalt anwendet. ${ }^{124}$

Dieser pauschalen ${ }^{125}$ Vorgehensweise ist entgegenzutreten. Die Vorstellung, dass die in der Beziehung übliche Sexualpraktik in einer Vergewaltigungssituation vom Opfer als weniger demütigend empfunden werde und der Täter hierbei auch geringeres Unrecht verwirkliche, geht fehl. Sie verkennt, dass sich das Unrecht einer Vergewaltigung nicht in der Vornahme eines körperlichen Akts erschöpft, sondern gleichzeitig das Innerste eines Menschen, den unantastbaren Intimbereich, in erniedrigender Weise in hohem Maße verletzt. Der in diesen Konstellationen regelmäßig erfolgende Vertrauensbruch sollte strafschärfende Berücksichtigung finden. ${ }^{126}$ Die gegenwärtige Praxis steht konträr zum sonstigen Umgang mit diesem Umstand im Besonderen Teil des StGB. Im Rahmen der Unterschlagung, § 246 II StGB, wird das Merkmal des Anvertrautseins qualifizierend berücksichtigt. Der Misshandlung von Schutzbefohlenen, § $225 \mathrm{StGB}$, die eine qualifizierte Körperverletzung darstellt, liegt ebenfalls der Gedanke des besonderen Vertrauensbruchs zu Grunde. Eine weit verbreitete Auffassung sieht des Weiteren gerade im Vertrauensbruch das kennzeichnende Merkmal des Heimtückebegriffs, wobei dessen Vorliegen in ,,institutionalisierten Vertrauensverhältnissen“ zur Bejahung des Mordmerkmals führen soll. ${ }^{127}$ Schließlich ist zu betonen, dass aus der Eingehung einer Ehe oder einer sonstigen Beziehung weder eine Verpflichtung zu regelmäßigen sexuellen Interaktionen folgt noch eine Durchsetzung derselben mit den Mitteln des $\S 177$ StGB als Selbsthilfe bzw. als minder schwer bagatellisiert werden darf.

\section{Vergewaltigung von Prostituierten}

Prostituierte genießen weiterhin nur eingeschränkten Strafrechtsschutz. ${ }^{128}$ Dazu der 5. Strafsenat des BGH : 129 „Im kriminologischen Gesamtspektrum der - auch qualifizierten - Vergewaltigungstaten besteht eine Polarität und ist dementsprechend bei der

2. Hs. StGB angenommen hatte, dies wurde vom BGH gerügt, da die Tat Bestrafungscharakter hatte und sich über mehrere Stunden hinzog.

123 BGH StV 2001, 453.

124 BGH StV 2001, 453.

125 Auch Schroeder, JZ 1999, 829 bewertet die meisten Beziehungstaten pauschal als ,zweifelhafte Fälle“.

126 Auch Hillenkamp, StV 1986, 150 ff., 154 regt an, in Fällen der Vertrauensvorgabe an eine Strafschärfung zu denken.

127 Küper, Strafrecht Besonderer Teil, 2008, S. 199.

128 Dazu auch Gaede, NStZ 2001, $238 \mathrm{ff}$.

129 BGH NStZ 2001, 29: Der ... Angekl. bestellte sich über eine Agentur telefonisch eine Prostituierte in seine Wohnung, um sich sexuelle Dienste kostenlos, gegebenenfalls unter Anwendung von Gewalt, gewähren zu lassen. Es erschien die Nebenklägerin, die zunächst eine Entgeltzahlung i. H. von 200 DM im Voraus verlangte. Durch erhebliche Schläge, Bedrohung mit einem Teppichmesser und das Ausreißen von Haarbüscheln erzwang der Angekl. im Wechsel zweimal ungeschützten Oralverkehr (fellatio), zweimal Beischlaf unter Verwendung eines Kondoms und schließlich schmerzhaften Analverkehr, bei dem er der Nebenklägerin ein Kissen in das Gesicht drückte. Zum Samenerguss kam es nicht. Schließlich zwang der Angekl. die Nebenklägerin zur Säuberung des WC; ebenso BGH NStZ 2001, 369. 
Strafzumessung eine Differenzierung geboten zwischen Taten gegen Frauen, die sich dem Täter zu - gegebenenfalls entgeltlichen - sexuellen Handlungen anbieten, und Taten gegen Opfer, die dem Täter keinerlei Anlass zu der Annahme geben, sie wären zu sexuellem Kontakt bereit ...". Der 2. und 3. Strafsenat erheben gegen diese Vorgehensweise Bedenken. ${ }^{130}$

Es muss nicht betont werden, dass die Rechtsprechung des 4. und 5. Strafsenats ${ }^{131}$ zur Vergewaltigung von Prostituierten unhaltbar ist. ${ }^{132}$ Antiquierte Erwägungen zum Zusammenhang zwischen einer Tätigkeit, die der traditionellen sexuellen Moral widerspricht, und einer daraus folgenden „Minderfähigkeit“ vergewaltigt zu werden, sind in höchstem Maße diskriminierend und sollten nicht mehr Ausgangspunkt gegenwärtiger juristischer Überlegungen sein. ${ }^{133}$

\section{Sexuelle Tatprovokation}

Auch vorangegangene Intimitäten im Sinne einer sexuellen Tatprovokation werden weiterhin strafmildernd berücksichtigt. Dazu der 5. Strafsenat des BGH: ${ }^{134}$ „Trotz Vorliegen eines Regelbeispiels des $\S 177$ Abs. 2 StGB kommt die Anwendung des Normalstrafrahmens des $\S 177$ Abs. 1 StGB bzw. die Annahme eines minder schweren Falls i.S.d. $§ 177$ Abs. 5 StGB in Betracht, wenn der Täter bei dem erzwungenen Vaginalverkehr nur sehr geringe Gewalt ausgeübt hat und das Tatopfer durch vorangegangenes Verhalten, das in dem Angeklagten den begründeten Eindruck erwecken konnte, das Tatopfer werde (weiteren) sexuellen Handlungen zustimmen, nicht unerheblich (wenn auch unbeabsichtigt) zur Entwicklung des Geschehens beigetragen hat. Dass der Angeklagte sich bis kurz vor der Tat noch Hoffnungen auf weiterführende intime Kontakte mit der Nebenklägerin machte, bleibt auch angesichts des Umstands, dass sie ihn zunächst nicht in die Wohnung lassen wollte, jedenfalls nachvollziehbar.... Angesichts des Gewichts der Milderungsgründe und der Tatsache, dass die Nebenklägerin selbst ihr Verhalten während des Tatgeschehens nachträglich als nicht wehrhaft und energisch genug einschätzt,..."

Interessant wird diese Urteilsbegründung, wenn man sich mit dem zugrundeliegenden Sachverhalt des Landgerichts auseinandersetzt. Dabei fällt auf, dass der BGH diesen Sachverhalt verzerrt ${ }^{135}$ und maßgebliche Gesichtspunkte unerwähnt lässt, um den Täter in ein besseres Licht zu rücken. So verschweigt der BGH u. a., dass der Angeklagte als Trainer von Ringern der Geschädigten, die eine zierliche Gestalt hat, definitiv körperlich überlegen war. Er betont, dass die Geschädigte und der Angeklagte in einem Lokal in der Tatnacht Zärtlichkeiten ausgetauscht hätten, verschweigt aber, dass die Geschädigte kein Interesse an einer weiteren Intensivierung hatte (der BGH wählt die Worte, sie habe

130 BGH NStZ-RR 2000, 358 Nr. 36; BGH NStZ 2001, 646.

131 BGH-Beschluss, 18.4.1973, 4 StR 135/73; BGH StV 1995, 635; BGH StV 1996, 26 f.; BGH NStZ 2001, 29; BGH NStZ 2001, 369.

132 Ebenso Hörnle, StV 2001, 454 ff., MüKo-Renzikowski, 2005, § 177 Rn. 65 Fn. 222 („Fragwürdige Strafzumessungserwägungen“) und Fischer, 57. Auflage (2010), § 177 Rn. 70.

133 Dazu auch Baurmann, Sexualität, Gewalt und psychische Folgen, 1996, S. 475.

134 BGH StV 2008, 81.

135 In seiner Anm. BGH StV 1986, 150 ff. kommt Hillenkamp zum selben Ergebnis. 
dies dem Angeklagten ,,bewusst verborgen gehalten“). ${ }^{136}$ Dies zeigte sich schon daran, dass sie bei der Taxifahrt nach Hause (der Angeklagte wollte ein Taxi teilen) im Wagen vorne Platz nahm. Des Weiteren ließ die Geschädigte den Angeklagten nur deswegen in ihre Wohnung, weil dieser sie bedrängte hatte, dass er die Toilette aufsuchen müsse. Der Annahme, dass „nur sehr geringe Gewalt“"137 ausgeübt wurde, ist auf Grund der Tatsache, dass der Angeklagte die Geschädigte mit seinem Körpergewicht von oben auf die Matratze drückte, entgegenzutreten. Die Ausführung zum Abwehrverhalten der Geschädigten entnahm der BGH einem Gespräch mit ihrem Therapeuten nach der Tat. Dort sagte sie, „sie habe das Gefühl, sie hätte sich bei dem Vorfall stärker wehren müssen, sie sei dazu aber nicht in der Lage gewesen“. ${ }^{138}$ Diese Art von Selbstvorwürfen findet sich häufig bei Vergewaltigungsopfern. ${ }^{139}$

\section{Abschließende Stellungnahme}

Die Täter-Opfer-Beziehung stellt insgesamt betrachtet geradezu „ein Leitmerkmal“140 der Abgrenzung des minder schweren vom normalen Fall der Vergewaltigung dar. ${ }^{141}$ Hervorzuheben ist, dass sich dieses Element bei den minder schweren Fällen des einfachen und schweren Raubes, $\S \S 249$ II StGB und $\S 250$ III StGB, nur sehr selten findet. Dies ist erstaunlich, da auch beim Raub eine Interaktion zwischen Täter und Opfer stattfindet. ${ }^{142}$

Das Institut eines minder schweren Falls ist grundsätzlich zu begrüßen, da dies die nötige Flexibilität bei der Strafzumessung schafft. Kritisch zu sehen ist jedoch die Praxis, eine Vergewaltigung über Abs. 1 zu einer sexuellen Nötigung und schließlich über Abs. 5 letztendlich zu einem minder schweren Fall einer sexuellen Nötigung herabzustufen. ${ }^{143}$ Sowohl der Handlungs- als auch der Erfolgsunwert einer Vergewaltigung lassen die Anwendung eines minder schweren Falls über Abs. 5 in den meisten Fällen als unangemessen erscheinen. ${ }^{144}$ Für die Anwendung des Sonderstrafrahmens des $\S 177$ V StGB ist es ja gerade nicht ausreichend, dass das Tatbild vom Durchschnitt der das Regelbeispiel verwirklichenden Sachverhalte abweicht; ,es muss sich vielmehr um einen Fall handeln, der bei einer Gesamtwürdigung von Tat und Täter auch mit dem

136 BGH StV 2008, 81.

137 BGH StV 2008, 81.

138 Urteil des LG Berlin vom 11.12.2006 - (506) 70 JS 624/06 (21/06).

139 Dazu Weis, Die Vergewaltigung und ihr Opfer, 1982, S. 81.

140 Albrecht, Strafzumessung bei schwerer Kriminalität, 1994, S. 307.

141 Vergewaltigungen finden zu 2/3 aller Fälle im sozialen Nahbereich statt; dazu Jäger, Das staatsanwaltliche Sonderdezernat „Gewalt gegen Frauen“, 2000, S. 87 f. Fn. 202.

142 Ebenso Hauch, ZfS 1988, 266; schlägt man z.B. Sch/Sch-Lenckner/Perron/Eisele, 27. Auflage (2006), § 177 Rn. 33 auf, so fällt einem die fast ganzseitige Kasuistik zum minder schweren Fall der Vergewaltigung (nur zu Abs. 1!) ins Auge; im Gegensatz dazu finden sich bei $\S 249$ Rn. 12 diesbezüglich nur zwei Zeilen; in § 250 Rn. 37 finden sich längere Ausführungen, die allerdings nur einen einzigen Entscheidungsnachweis zu einer Tatprovokation durch das Opfer (BGH StV 1982, 575) enthalten.

143 Die Rechtsprechung bleibt freilich bei dem Ausdruck ,,minder schwerer Fall einer Vergewaltigung".

144 Ebenso MüKo-Renzikowski, 2005, § 177 Rn. 110. 
durchschnittlichen Erscheinungsbild und Unwertgehalt einer sexuellen Nötigung i. S. d. Abs. 1 nicht mehr vergleichbar ist". 145

Daneben ist es äußerst bedenklich, dass sich Strafzumessungserwägungen und insgesamt das Verständnis von einer Vergewaltigung weiterhin an überkommenen Vergewaltigungsmythen und -klischees orientieren. ${ }^{146}$

Dazu zählt die Bedeutung, die den Widerstandshandlungen beigemessen wird. ${ }^{147}$ Hierbei treten die überholte Vorstellung von einer vis haud ingrata und der Anspruch, dass sich eine Frau gegen eine „echte“ Vergewaltigung stets unter Einsatz all ihrer Körperkräfte wehren muss, zutage. Im Kommentar Schönke/Schröder finden sich unter Ziff. III zu $§ 177$ StGB Ausführungen zum Vorsatz des Täters. Dabei heißt es: „Der Vorsatz muss sich ferner insbesondere darauf erstrecken, dass das Opfer der Tat ernsthaften Widerstand entgegensetzt ${ }^{\text {“ }}{ }^{148}$ Natürlich muss der Täter erkennen, dass das Opfer nicht mit den sexuellen Handlungen einverstanden ist. Neben verbalen Ablehnungsäußerungen ist auch Widerstand Ausdruck des Nicht Einverstanden Seins mit dem Vorgehen des Täters. Das Adjektiv ,ernsthaft“ ist jedoch verfehlt, da jeder Widerstand auch Widerstand im wahrsten Sinne des Wortes und Tuns bedeutet. Jedes zusätzliche Deutungsmuster hinsichtlich einer Ernsthaftigkeit ist dabei fehl am Platze.

Die Vergewaltigungstat wird weiterhin zu maßgeblich von der Vorstellung bestimmt, dass es sich um ein Trieb- und Spontandelikt handelt. ${ }^{149}$ Diese These wurde jedoch durch psychotherapeutische Untersuchungen widerlegt ${ }^{150}$ und ist in der kriminologischen Forschung überholt. ${ }^{151}$ Die Vergewaltigungstat ist ein sexueller Gewaltakt, der die Unterwerfung und Demütigung des Opfers intendiert. ${ }^{152}$ Man bedenke hierbei auch den Einsatz der Vergewaltigung als Kriegswaffe. ${ }^{153}$ Die Vorstellung von einem krankhaften Triebtäter, der sich seinen Hormonen unterwerfen muss, ist obsolet. ${ }^{154}$

Der geschlechtsspezifischen Situationsverkennung als Strafmilderungsgrund wird zu viel Platz eingeräumt ${ }^{155}$ bzw. unterliegt einer falschen Interpretation. Ausgangspunkt dieser These von Schorsch ist, dass Frauen und Männer die Wirklichkeit unterschiedlich konstruieren. ${ }^{156}$ Der Mann deutet eine neutrale Freundlichkeit der Frau als Verliebtheit,

145 Sch/Sch-Lenckner/Perron/Eisele, 27. Auflage (2006), § 177 Rn. 33.

146 Schneider, FS Lenckner, 1998, 847 ff., 856 führt aus, dass die vergewaltigungsunterstützenden Stereotypen in starken gesellschaftlichen Minderheiten vertreten werden.

147 BGH StV 2008, 81.

148 Sch/Sch-Lenckner/Perron/Eisele, 27. Auflage (2006), § 177 Rn. 13.

149 Baurmann, Sexualität, Gewalt und psychische Folgen, 1996, S. 474 f.; Weis, Die Vergewaltigung und ihr Opfer, 1982, S.20: er belegt, dass die Mehrzahl der Vergewaltigungen im voraus entweder vollständig oder teilweise geplant werden; ebenso Butzmühlen, 1978, S. $89 \mathrm{f}$.

150 Dazu Hauch, Gelebte Sexualität im Maßregelvollzug?, 2006, S. 18 ff., S. 19.

151 Schneider, FS Lenckner, 1998, S. 847; Schorsch, Sexualstraftäter, 1971, S. 80 belegt, dass $88 \%$ der Notzuchtstäter stark aggressive Täter sind.

152 MüKo-Renzikowski, 2005, § 177 Rn. 9; in BT-Drs. 13/323, S. 5 heißt es folglich, dass es sich bei der Vergewaltigung um eine „,brutale Erniedrigung“ handelt.

153 Dazu Greve, Vergewaltigung als Völkermord, 2008, S. 27 ff.; Burkhardt, Verbrechen gegen die Menschlichkeit, 2000.

154 Schneider, FS Lenckner, 1998, 847.

155 Schneider, FS Lenckner, 1998, 866: er hebt hervor, dass dieser Theorie in der viktimologischen Diskussion zu viel Bedeutung beigemessen wurde.

156 Schorsch, Sexualstraftäter, 1971, S. 214 f. 
so dass eine projektive Verkennung der Situation entsteht, die nicht selten auch den sexuellen Vollzug überdauert. Laut der empirischen Untersuchung von Jäger ${ }^{157}$ zählten zu den häufigsten Faktoren der Tat Formen dieser Situationsverkennung, nämlich u. a. ein ,vermeintliches Recht auf Grund eines bestehenden Intimverhältnisses, die Hoffnung, das Opfer werde zum Geschlechtsverkehr bereit sein, ein vorangegangener Flirt, unvorsichtige oder Hoffnung machende Gesten oder Bemerkungen des Opfers, aufreizende Auffälligkeiten des Opfers“. ${ }^{158}$ Treffen diese Faktoren mit geringer körperlicher Gegenwehr von Seiten des Opfers und als „unbrutal“ eingeschätzte Gewalt von Seiten des Täters in der Tatsituation zusammen, wird zum einen schon bezweifelt, dass überhaupt eine echte Vergewaltigung vorliegt ${ }^{159}$ und zum anderen Verständnis für den Täter gezeigt, da aus seiner Sicht eine von sexuellen Intentionen geprägte Situation vorlag. ${ }^{160}$ Die geschlechtsspezifische Situationsverkennung gibt damit opferfeindlichen Perspektiven Raum. Gerade die Viktimologie zur Vergewaltigung ist durch diese Perspektive in großem Maße geprägt. ${ }^{161}$ Vorsicht ist jedoch angebracht, da dem Täter Schutzbehauptungen ermöglicht werden, die den Rechtsbruch neutralisieren sollen. ${ }^{162}$ Androzentrische Sichtweisen und eine selektive Wahrnehmung gestalten eine Vergewaltigungstat so nachträglich in eine Verführungssituation um. ${ }^{163}$

Spiegelbildlich gesehen hängt damit die Lehre vom Mitverschulden des Vergewaltigungsopfers zusammen. ${ }^{164}$ Es stellt sich die schwierige Frage, ob dieses in bestimmten Konstellationen für den Schutz seines Rechtsguts sexuelles Selbstbestimmungsrecht selbst verantwortlich ist. ${ }^{165}$ In der Rechtsprechung zum Betrug gibt es einige wenige Fälle, die es strafmildernd werten, dass das Opfer ,nachlässig“" gehandelt habe und der Täter infolgedessen zur Tatbestandsverwirklichung lediglich geringe kriminelle Energie einsetzen musste. ${ }^{166}$ Beim minder schweren Fall des schweren Raubes $§ 250$ III StGB findet sich eine Entscheidung, die eine Tatprovokation bejaht. ${ }^{167}$ Der Vergleich mit anderen Delikten ist demnach nicht weiterführend. Hillenkamp liefert plausible Ansät-

157 Jäger, Das staatsanwaltliche Sonderdezernat „Gewalt gegen Frauen“, 2000, S. 72 f.

158 Jäger, Das staatsanwaltliche Sonderdezernat „Gewalt gegen Frauen“, 2000, S. 73.

159 Dazu Weis, Die Vergewaltigung und ihr Opfer, 1982.

160 Zuletzt BGH StV 2008, 81; dazu auch Baurmann, Sexualität, Gewalt und psychische Folgen, 1996, S. 319.

161 Weis, Die Vergewaltigung und ihr Opfer, 1982, S. 17; vgl. Schneider, Viktimologie, 1975, S. $118 \mathrm{ff}$. (Schneider scheint seine frauenfeindlichen Thesen jedoch überwunden zu haben: vgl. Schneider, FS Lenckner, 1998, 847 ff.); Amir, Patterns in Forcible Rape, 1971, S. 336 f.

162 Schneider, FS Lenckner, 1998, 861.

163 So auch Hillenkamp, StV 1986, 152.

164 Dazu die umfassende Untersuchung von Weis, Die Vergewaltigung und ihr Opfer, 1982, S. 81.

165 Hier kann nicht der Frage nachgegangen werden, ob ein bestimmtes Opferverhalten bereits zum Ausschluss des Tatbestandes oder der Rechtswidrigkeit führen kann. Die h. M. in Rechtsprechung und Literatur lehnen dies ab: Roxin, Strafrecht AT I, 4. Auflage (2006), $\S 14$ Rn. 15 ff. m. w. N.; Hillenkamp, Vorsatztat und Opferverhalten, 1981; vgl. zur Viktimologie, die seit Ende der 1970iger Jahre diskutiert wird u. a. die soeben zitierten sowie Schünemann, ZStW 1978, 11 ff.; ders., in Schünemann/Dubber, Die Stellung des Opfers im Strafrechtssystem, 2000, S. 1 ff.

166 BGH StV 1983, 326; BGH wistra 1986, 172.

167 BGH StV 1982, 575. 
ze. ${ }^{168}$ Eine strafmildernde Opferpartizipation soll erst dann eintreten, wenn sich das erwachsene und voll einsichtsfähige Opfer trotz wahrgenommener evidenter Gefahranzeichen, weil der Täter sein sexuelles Ansinnen eben gerade nicht verbirgt, dem Täter ausliefert. ${ }^{169}$ Unter diesem Blickwinkel müssten zahlreiche Urteile revidiert werden. Sie befreit die Vergewaltigungstat von antiquierten Erklärungsmodellen und -mythen.

Zuletzt ist - da es die konservative Verhaftung verdeutlicht - besonders hervorhebenswert, dass das sexuelle Selbstbestimmungsrecht in $\S 177$ StGB nur dann als schützenswert angesehen wird, wenn die körperliche Unversehrtheit betroffen ist. ${ }^{170}$ Es findet auch - soweit mir ersichtlich - keinerlei Diskussion über diesen Umstand statt. Die gegenwärtige juristische Bewertung eines Sachverhalts als Vergewaltigung hat sich damit nicht weit von der des Mittelalters entfernt. ${ }^{171}$

\section{Fazit}

Der Vergewaltigungstatbestand hat erst seit 1997 eine „bewegte“ Geschichte. ${ }^{172}$

Der Umgang mit diesem erfordert ein sensibles und reflektiertes Geschichtsbewusstsein. Des Weiteren den Willen und den Mut, den Vorgang einer sexuellen Nötigung / Vergewaltigung einer vorurteilsfreien Revision zu unterziehen.

168 Hillenkamp, StV 1986, 153.

169 Hillenkamp, StV 1986, 154.

170 Dabei finden sich im common law Rechtsordnungen, die ein Vorgehen ,gegen den Willen“ des Opfers für das Vorliegen einer Vergewaltigung ausreichen lassen; vgl. auch den Reformvorschlag der Grünen in BT-Drs. 10/6137, S. 36; BT-Drs. 12/3303, S. 7.

171 Eine umfassende Stellungnahme ist mir hier aus Platzgründen nicht möglich.

172 Die Einrichtung von Sonderdezernaten „Gewalt gegen Frauen“, die seit Ende der 80iger Jahre in den meisten Bundesländern eingerichtet wurden, ist positiv hervorzuheben; dadurch kann der sekundären Viktimisierung entgegengetreten werden: dazu Baurmann, Sexualität, Gewalt und psychische Folgen, 1996, S. 475; der Vorschlag, Sonderzuständigkeiten für Sexualdelikte auch an den Gerichten einzurichten, hat sich in der Reformdiskussion leider nicht durchgesetzt: dazu der SV Helmken, 35. Sitzung des Rechtsausschusses des Bundestags, 6.12.1995, S. 9 ff. 